\title{
Chapter 6 \\ Participatory Aspects of ICT \\ Infrastructures for Cancer Management
}

\author{
Haridimos Kondylakis, Lefteris Koumakis, Ketti Mazzocco, \\ Manolis Tsiknakis, and Kostas Marias
}

\section{Introduction}

As cancer research has come up with new, more effective treatments more and more cancer patients are being cured, and very many more enabled to live with their cancer. The disease is now frequently managed as a chronic illness requiring long-term surveillance and, in some cases, maintenance treatment. Cancer care occurs on a continuum that stretches from prevention to the end of life, with early detection, diagnosis, treatment, and survivorship in between. This implies a transformation in the nature of the existing healthcare model from reactive to preventive, and to personalized medicine. As a chronic illness, however, there is an urgent economic and pragmatic need for patients and families to manage their own care, and for the healthcare system to develop efficient strategies in supporting the achievement of this objective. Self-management support is defined as "what health services do in order to aid and encourage people living with a long term condition to make daily decisions that improve health related behaviours and clinical and other outcomes'. Educating patients to self-management of disease strengthens health behaviours by promoting health literacy and collaborative decision-making skills, problem solving and action planning related to their condition. Such an approach is being embraced

H. Kondylakis $(\bowtie) \cdot$ L. Koumakis $\cdot$ M. Tsiknakis $\cdot$ K. Marias

Computational BioMedicine Laboratory, Institute of Computer Science,

Foundation for Research and Technology, Crete, Greece

e-mail: kondylak@ics.forth.gr; koumakis@ics.forth.gr; tsiknaki@ics.forth.gr;

kmarias@ics.forth.gr

K. Mazzocco

Department of Oncology and Hemato-Oncology, University of Milan, Milan, Italy

Applied Research Division for Cognitive and Psychological Science,

European Institute of Oncology (IEO) IRCCS, Milan, Italy

e-mail: ketti.mazzocco@ieo.it 
by government policies and in clinical practice, as demonstrated by the increasing number of initiatives and trials for patients' self-management.

Advances in information and communication technology (ICT), together with the recent spread of portable devices such as smartphones and tablets, offer the opportunity to re-design self-management. ICT can provide the means to transform the role of the patient from a passive recipient of healthcare services to an active, informed participant of medical decision-making processes in charge of his or her own well-being.

In this chapter, we review how this vision of building ICT platforms that promote and enhance the participation of all stakeholders involved in cancer management has been implemented in the context of five European research projects. Primarily they focus on the individual patient, empowering them and enabling better selfmanagement, but also on the various care providers and health experts involved in the patient journey. Those projects focus on the transformation of the paternalistic model of patient-doctor to a new model that promotes the participatory aspects of all involved participants. We present achievements in the area of those five EU proposals, we identify the solutions provided and we discuss lessons learnt. Then we summarize and provide guidelines on the development of future ICT platforms.

The rest of this chapter is structured as follows: In Sect. 2, we elaborate on participatory aspects for cancer management and we highlight the need to transform the existing model, discussing also recent evidences on the area. Then in Sect. 3 we present experiences from five relevant EU projects (iManageCancer, MyHealthAvatar, p-Medicine, EURECA and INTEGRATE) and we elaborate on their approach to enable patients to participate in the management of their disease. Then in Sect. 4 we summarize the findings and we present directions for the development of future ICT platforms with a strong focus on further enhancing the patient participation on the management of their disease.

\section{Participatory Aspects for Cancer Management}

\subsection{From Personalized to Participatory Medicine}

Since the early 2000s, we are observing a profound revolution in the healthcare, with a shift in the medicine approach toward a predictive, preventive and personalized one, slowly moving towards the so-called 3P model (Hood et al. 2004; Weston and Hood 2004). Personalized medicine refers primarily to the genomic and molecular biology. Thanks to a unique combination of biological information, it is possible to design new pharmacological therapies tailored to the specific molecular picture of the patient.

Despite the important changes of this new approach, the underlying paradigm considered the patient still as a passive recipient of care, with the healthcare professionals as main actors. Three main aspects make this perspective suboptimal:

1) Medicine normally acts under uncertainty conditions, and some relevant information lies locked within the patient's body (Sox et al. 2013). To reduce uncertainty, besides the clinical examinations, some data can be obtained only from 
the patient: doctors must rely on what the patient reports during the visits or using the patient reported outcomes measures (PROMs). In line with this perspective, the need to consider patients' knowledge, experiences and needs is not a mere ethical position, but it has also clinical consequences. As shown by Stacchiotti and Sommer, patients are not only passive care receivers but special collaborators that proactively can help clinicians and researchers reduce the aforementioned uncertainty (Stacchiotti and Sommer 2015) and find new therapeutic solutions.

2) The last 40 years has seen another initially slow but important shift in medicine: from a biomedical to a bio-psycho-social paradigm (Engel 1977; Inerney 2018). The World Health Organization has indeed defined health as a state of complete physical, mental, and social well-being, emphasizing the shift from the dominant paradigm with a focus on the disease to the new paradigm of wellness as a whole (WHO). The disease cannot be considered in isolation from its host: there is the need to understand and treat the disease considering the patient as a system, where the single parts strictly interact and produce an outcome that is something more than the sum of the single parts. In this perspective, personal psychological characteristics and social contexts interact with the biological factors in affecting the clinical outcomes. Consequently, the health professionals' aim within this paradigm is not anymore just to cure and increase life expectation, but to guarantee a good quality of life. Beside objective outcomes, the attention is paid also to subjective outcomes. The participation of the patient becomes therefore necessary, since the evaluation of what a life of quality means depends on the person's subjective values, priorities and preferences.

3) The spread of technology has made it easier for patients to access health information through the Internet. The constant growth of Internet as a source of health information (Morahan-Martin 2004; Internet World Stats 2016) (independently on the quality of the information) has contributed to make the patient a 'clinical expert', changing his/her role from passive recipient to active agent that wants to know and wants to participate in the decisions relevant for his/her health.

All these factors pushed for integrating the P3 medicine approach with other Ps, in particular a fourth P, standing for participatory (Hood and Friend 2011), and a fifth P that stands for psycho-cognitive (Gorini and Pravettoni 2011). In other words, the need for actively involving the patient in the care pathway has become a moral imperative (Pravettoni et al. 2016).

\subsection{Critical Factors of Participatory Medicine}

The Society for Participatory Medicine defined participatory medicine as 'a movement in which networked patients shift from being mere passengers to responsible drivers of their health, and in which providers encourage and value them as full partners' (Frydman 2010). Individual's genetic, molecular, cellular, organ and social networks will be combined into an overall 'network of networks', to give a detailed 
picture of the normal and disease-perturbed states. Following this approach, healthcare and wellness is not restricted anymore within the hospitals but moves into foundations and the patients' home. Chordoma Foundation (http://www.chordomafoundation.org) is a strong example of the power that the patient can have to make the difference in research for the cure of cancer or other fatal diseases, transforming, for example as in the case of Chordoma, a rare cancer to a not-so-rare cancer. Thanks to technology, indeed, Sommer, the executive director of Chordoma Foundation and personally diagnosed with Chordoma cancer, rounded up patients and researchers working on that specific cancer, optimizing the resources earlier scattered around the world, increasing awareness of the problem and breaking down barriers to progress.

Patients' participation in healthcare is relevant not only for improving research on new cancer treatments, but has positive effects on the patient's health condition. Barnato et al. (2007) noted that 'in an ideal world [...] patients would come to a cancer consultation armed with sufficient knowledge, clarity about their personal value, and the ability to engage in a thoughtful discussion about the pros and cons of treatment options. Providers, in turn, would be prepared to support their patients, armed with an understanding of the patient's knowledge gaps, personal values about possible outcomes and treatment preferences' (p. 627). The nature of malignant diseases such as cancer requires patients to learn about and comprehend the illness, make difficult decisions regarding ensuing treatment, and cope with the consequences of the illness. It has been found that having relevant information not only helps cancer patients to understand the disease, but it also facilitates their decision making and coping with the disease. Especially with cancer becoming a chronic disease, treatment places new demands on patients and families to manage their own care. A collaborative and interactive relationship between patients and health professionals can empower patients to take on responsibility for their condition with the appropriate clinical support. In this new concept of healthcare, clinician and patient are part of the same team: patients are empowered by more available information, and take a more active and responsible role, while clinicians welcome them as knowledgeable partners in clinical practice. The value of co-participation is particularly evident in the short- and long-term outcomes of shared decision making (Kane et al. 2014). Among the short-term outcomes are: satisfaction with and confidence in the made decision, satisfaction with physician-patient relationship, trust in the physician, increase in patient's self-efficacy and improvement in physical and emotional well-being. Long-term patient's outcomes are increases in treatment adherence, remission and quality of life. Despite the added value of the participation of patient in the information exchange and on decision-making process, its implementation in clinical practice is however still low. A systematic review (Kane et al. 2014) found that decision aids (DAs) improve patients' knowledge, reduce decisional conflict and motivate people to take a more active role in decision making. Informed decision making should combine the patients' personal values and the best available data. However, many patients have difficulties in associating these two components. Support tool such as a prompt list or decision aids can help them better manage the situation (Brown et al. 2013). However, DAs are usually 
standardized and are not necessarily adapted to the psycho-emotional state of the patient (Bekker et al. 2003; Davis et al. 2014).

\subsection{Psychological Factors as Moderators of Participatory Medicine}

In order to make the participatory approach efficient, we should open up space to explore patients' personal psychological, cognitive and social (familial) state (Gorini and Pravettoni 2011; Pravettoni and Gorini 2011). All these factors act as barriers or facilitators in patient's self-management.

Dealing with health information and decisions is not a straightforward process, especially if we consider that health literacy is generally poor among the population. Several studies have demonstrated that terminology is a barrier that decreases the level of engagement in the healthcare pathway (Keselman and Smith 2012) because of a poor understanding of medical documents, a high difficulty in adding non-understandable terms (their personal medical information) in Personal Health Records (PHR) (Genitsaridi et al. 2013, 2015), the discomfort in communicating with the physician about something they do not completely understand.

A first step in this direction is to understand how patients process information and what factors affect their capability to adapt and manage their illness and the decisions related to treatment, adherence, and lifestyles. The information selection and interpretation strongly depend on the patient's status quo, including knowledge, values, needs, believes and emotions, where emotions have a fundamental role in guiding the search for information and therefore the construction of preferences (Pravettoni et al. 2016b; Gorini et al. 2014).

In line with the aforementioned bio-psycho-social approach, besides a focus on the biological and psychological individual characteristics, it is important to consider social dynamics that can affect patient's self-management. The impact of a cancer diagnosis is indeed not limited to the individual; rather it influences their family and social network, which in turn can affect the patient's psychological state and therefore their empowerment. According to the family systems theory, a change in one member of the system affects the whole system (Von Bertalanffy 2003). In presence of an inadequate readjustment to the trauma, the system can develop clinically significant levels of distress, higher risk of developing psycho-social problems, high levels of conflict and low family cohesion (Van Schoors et al. 2015). The level and type of family adaptation eventually affects the patient's ability to cope with the illness.

The challenge of research in the last years, in line with the P5 approach, has been to build supportive environments that could be personalized for the specific patient. The multidisciplinary collaboration between oncologists, psychologists, engineers, IT professionals allowed the progress in the development of e-tools which can enhance patient empowerment and self-management, being also time-efficient and thus more easily integrated with the current clinical routine. What seemed an ideal in the traditional clinical practice becomes possible in the 'virtual' eHealth environ- 
ment: to integrate all patient's data to provide a real personalized medical service. Coherently, information provided by physicians are tailored on the patient's psychocognitive profile; decision aids can support patient's choice according to his/her preferences and values, and that facilitate physician-patient shared decision making; specific smart applications support the patient to cope with cancer, manage their condition and adhere to healthy lifestyle; alerts from eHealth platforms inform physicians about clinical and psycho-emotional states of the patients.

\section{ICT Solutions from Relevant EU Projects}

\section{1 iManageCancer}

The iManageCancer project with the subtitle 'Empowering patients and strengthening self-management in cancer diseases', aims to provide a cancer disease selfmanagement platform designed according to the specific needs of patient groups and focusing on the well-being of the cancer patient with special emphasis on psycho-emotional evaluation and self-motivated goals, as P5 approach shows. The platform is centred on a Personal Health Record that exploits recent advances on Health Avatars for the individual cancer patient surrounded by mHealth applications designed to encourage the patient, enhance clinician-patient communication, maximize compliance to therapy, inform about drug interactions, and contribute to the management of pain and other side effects of cancer treatment.

\subsubsection{Technological Contribution}

The iManageCancer platform was designed on clinical evidence and in close collaboration with clinical experts, IT specialists and patients and was assessed in clinical pilots with adult and paediatric cancer patients. The architecture of the technological contribution of the iManageCancer project is shown in Fig. 6.1.

Apart from the Personal Health Record that is the core component of the iManageCancer (Kondylakis et al. 2017a), the platform provides tools to assess adherence to therapy, physiological and psychological status and recommendations to the patient (Iatraki et al. 2018) according to his or her disease type and psycho-emotional status in order to promote a positive and healthier psycho-emotional state (Faccio et al. 2018) (Kazantzaki et al. 2016). The platform is further complemented by an expert system with formal self-management models oriented to decision support (Schera et al. 2018), serious games for children (Hoffmann and Wilson 2018) and adults (Zhang et al. 2018), e-Consent tool (Kondylakis et al. 2015c, 2017b) and anonymized data analysis (Koumakis et al. 2016b). 


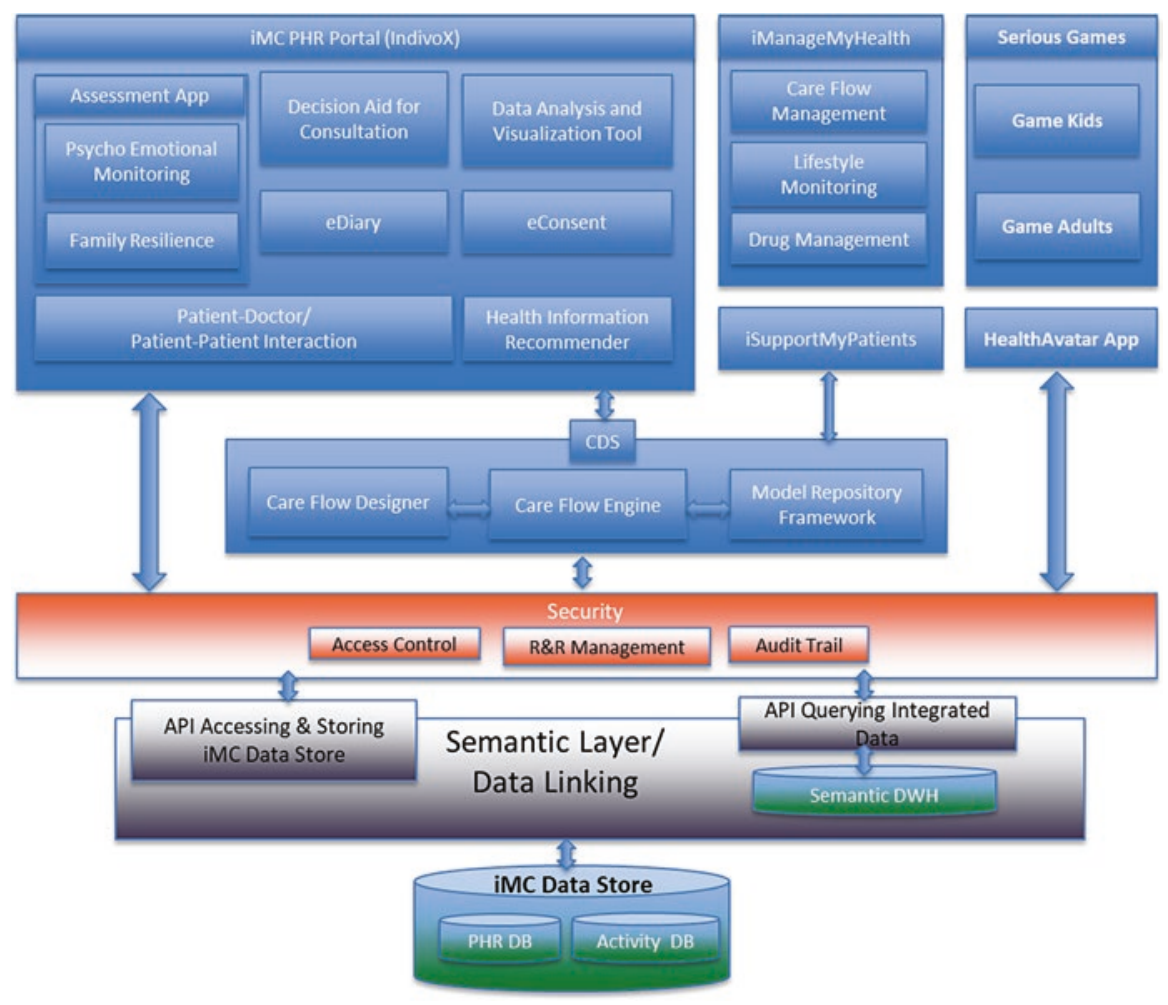

Fig. 6.1 The main components of the iManageCancer platform and their interactions

\subsubsection{Participatory Aspect of the Developed Solution}

iManageCancer aimed to arrange planned eHealth decision-making aids in cancer, promoting a self-aware and informed decision-making approach, compensating difficulties in shared decision-making approach with clinicians. In clinical practice, barriers in shared decision making are multiple. The most common ones are healthcare professionals' concerns about not having enough time, the perception that patient characteristics or the specific clinical situation were not conducive to shared decision making, the belief that some patients prefer a paternalistic approach without asking patients about their preferred role in decision making, and limited familiarity with shared decision making (Gravel et al. 2006). The proposed solution includes a novel approach for the collaborative management of cancer diseases with the informed and encouraged patient in a central role in the decision-making process.

Furthermore, the iManageCancer integrated mobile services act as the entry point for interactive disease self-management in close collaboration with the healthcare team. It advances disease management through reinforcement of the role of the patient in the management process, enabling better collaboration and interaction of 
informed patients with doctors, better planning of management processes and better compliance of patients to therapy through the mobile services of the platform.

Disruptive technologies for healthcare were also included such as serious games for monitoring the psychological dimensions of the disease. To this direction, games for children and adolescents but also for their relatives were developed and piloted for mobile platforms.

\subsubsection{Lessons Learnt}

While for several of the iManageCancer technological components there is already evidence that they can work for the benefit of the patient (e.g. psycho-emotional evaluation for improving therapy services etc.), the clinical pilots deployed in this project had to eventually face and overcome scepticism regarding the acceptance of such mHealth empowering technologies designed for the cancer patient. iManageCancer overcomes this obstacle by its serious commitment in the clinical pilots for paediatric oncology and adult oncology (prostate, breast and lung cancer) as well as the continuous focus on the cancer patient, offering technology for the best possible care, targeting on making cancer therapy a more personalized, continuous and participatory experience.

Another barrier to innovation in research projects relates to the unavailability of clinical data. There are usually significant complexities with respect to involving both clinicians and patients in real-life pilots. The large datasets available in the iManageCancer project and the real-life evaluations with patients and clinicians can eventually speed up innovation in areas such as data mining (Koumakis et al. 2018) and clinical decision support.

Results showed mixed evidences of improvements in patient empowerment due to lack of time and treatment-induced stress and psychological problems. Nevertheless, coping with cancer, mood and cancer resilience were improved for the trial arm using the platform. In addition, users recognized the usability and the usefulness of the developed platform. The different tools and services of the iManageCancer platform were developed and further optimized in several cycles implementing feedback of end-users and experiences from the pilots.

\subsection{MyHealthAvatar}

MyHealthAvatar project (http://www.myhealthavatar.eu/) (Kondylakis et al. 2015b; Maniadi et al. 2013) was an attempt to record digitally the health status of individual citizens. The goal was to create a digital representation of the user, a health avatar, acting as a mediator between the end-users and health-related data collections. It was designed as a lifetime companion for individual citizens that facilitates the collection, the access and the sustainability of health status information over the long term. 


\subsubsection{Technological Contribution}

The architecture of the technological contribution of the MyHealthAvatar project is shown in Fig. 6.2. It consists of the following layers: (a) the data repository, (b) the semantic integration layer, (c) the auditing service, (d) the layer for linking with external sources, (e) the MyHealthAvatar toolbox and (f) the GUI (graphic user interface) layer. The data repository includes a data lake with various data sources that are available to the project, a tool/model repository and an imaging repository. Selected data out of this data lake are extracted, transformed and loaded onto a Virtuoso triple store where they are integrated. Data can also be extracted from

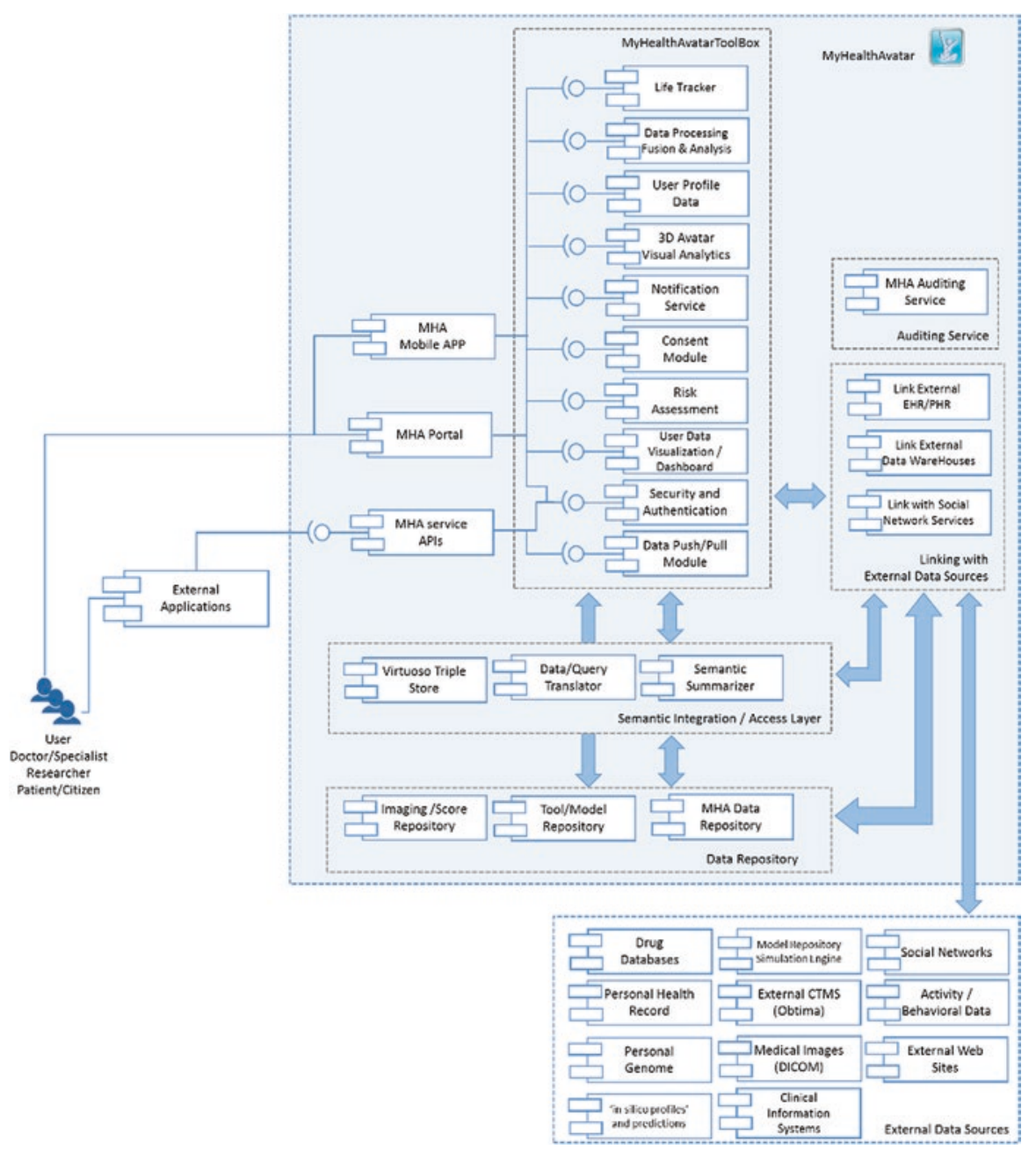

Fig. 6.2 The main components of the MyHealthAvatar platform and their interactions 
external data sources using the various linking services. In addition to these data, the MyHealthAvatar toolbox includes all necessary services and implements the business logic to be presented through the GUI layer either using the MyHealthAvatar portal or the MyHealthAvatar Mobile app. The GUI layer facilitates and encourages self-monitoring and self-management via a number of different approaches (semi/ automatic monitoring of individuals' steps count, calories consumption, active minutes, locations, movements, mood) through a 'virtual avatar', assists self-knowledge discovery through analysis and mining of personal health and activity data. Finally, it supports self-management of general health and well-being, and a range of chronic diseases via tailored intelligent tools (hypertension management, cardiovascular risk, diabetes, etc.). A specific branch of the platform (Zhang et al. 2018) is targeting lifestyle management support for cancer patients with all available tools.

\subsubsection{Participatory Aspect of the Developed Solution}

The MyHealthAvatar project focused on providing an ICT infrastructure for enhancing citizen's participation in self-management, disease prevention and patient empowerment as P5 suggests. It tried to lift the barriers primarily from the perspective of raising awareness, knowledge and motivation via risk appraisal and information provision. In addition, it provided tools to facilitate patients with chronic conditions to build and improve their health literacy and to provide a repository for recording medical, health, activity and diet information in the long term. Analyzing and mining those data, important events in personal life can be highlighted, personal life patterns can be identified and outliers can be detected such as sudden changes of lifestyles; allowing for self-assessment of health status; summarizing and reporting the performance of individual users over a certain period.

\subsubsection{Lessons Learnt}

In the process of building and piloting such a diverse platform, multiple lessons were learnt. For example, any platform trying to empower patients with chronic diseases should be validated in a real setting, involving multiple clinical partners and patients as well. Solutions 'one size fits all' are not appropriate in such a context and specific reconfiguration is needed for different types of diseases, as the information needs are different in each case.

In addition, the incorporation of health psychology models, is a key as they provide a foundation for health behaviour intervention. This enables the identification of key roles in patients' self-management, with a focus on their influence, and eventually on the compliance with medication plans, yielding individually tailored behaviour interventions to improve their compliance.

Finally, further investigation on usage of decision support tools is required. As gradually those tools mature, and their recommendations (Kondylakis et al. 2015a) are based on solid evidences, the confidence of the citizens will also increase enhancing their participation in their self-management. 


\section{3 p-Medicine}

p-Medicine (From data sharing and integration via VPH models to personalized medicine) tried to formulate an open, modular framework of tools and services for efficient, secure sharing and handling of large personalized datasets (Marés et al. 2014). The platform enables demanding Virtual Physiological Human (VPH) multi-scale simulations (in silico oncology), builds standards-compliant tools and models for VPH research and provides tools for large-scale, privacy-preserving data and literature mining, a key component of VPH research. The project ensures that privacy, non-discrimination and access policies are aligned to maximize protection of and benefit to patients. The p-Medicine tools and technologies were validated within the concrete setting of advanced clinical research. Pilot cancer trials were conducted, based on clear research objectives, emphasizing the need to integrate multi-level datasets, in the domains of Wilms tumour, breast cancer and leukaemia. To sustain a self-supporting infrastructure, realistic use cases were built, demonstrating tangible results for clinicians.

\subsubsection{Technological Contribution}

Figure 6.3 shows the main components and their interdependency of the pmedicine system architecture from a clinical perspective.

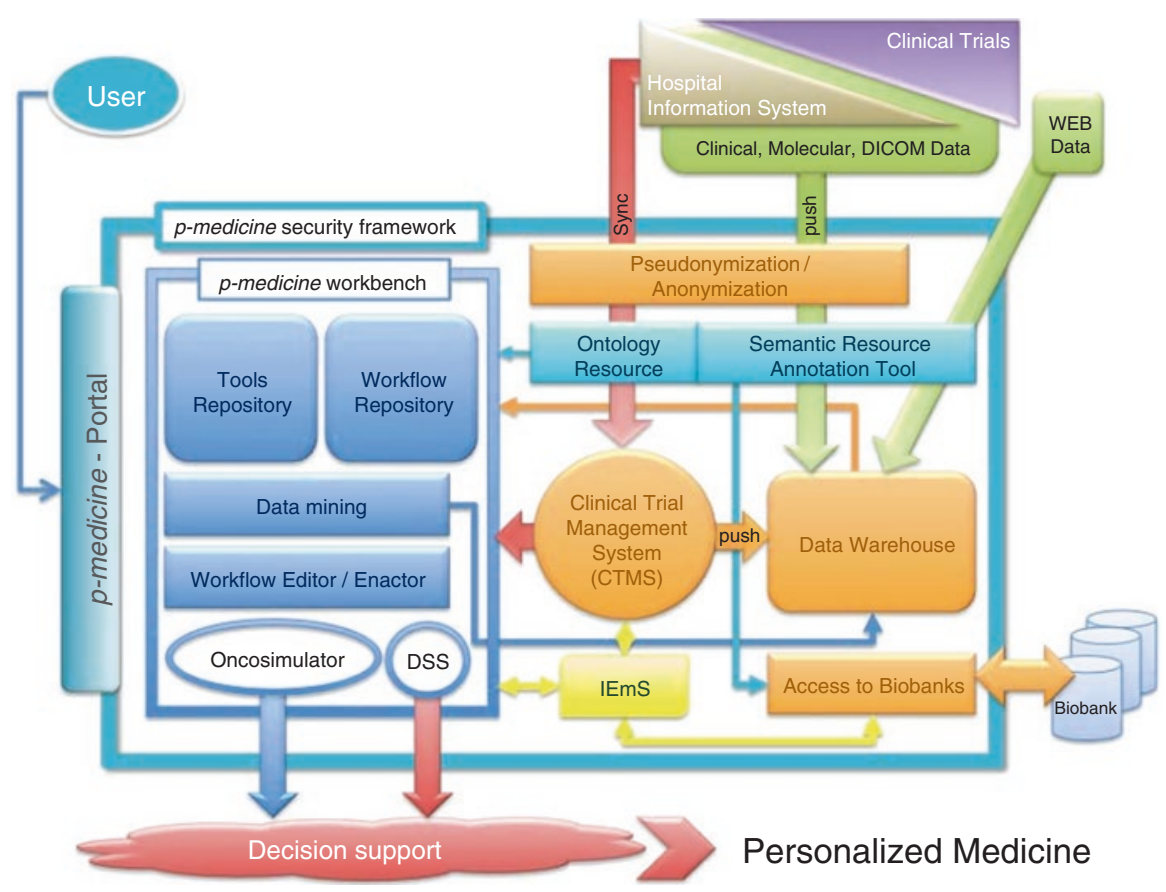

Fig. 6.3 The main components of the p-medicine platform and their interactions 
A user is able to get access to p-medicine via a secure portal to use tools and workflows from the p-medicine workbench to execute his or her models by mining data from the data warehouse. The data warehouse is fed by data from Hospital Information Systems (HIS) or the integrated Clinical Trial Management Systems (CTMS) via a push service. The CTMS can synchronize with the HIS using a sync service. Data entering the p-medicine environment are pseudonymized/anonymized and semantically annotated (Sfakianaki et al. 2015). Access to external biobanks can be established and freely available data from the web can be stored in the data warehouse with the aid of literature mining (Potamias et al. 2005). Depending on the scenario, users are able to execute models (Sfakianakis et al. 2009), use the p-medicine Oncosimulator (Stamatakos et al. 2014), systems biology models (Koumakis et al. 2016a; Koumakis et al. 2017; Mehta et al. 2016) or they can use the Decision Support System (Bucur et al. 2016). In all cases results lead to personalized medicine via decision support. Patients as users of p-medicine can interact with the p-medicine environment via IEmS (Kondylakis et al. 2012), a collaborative environment for patient empowerment.

\subsubsection{Participatory Aspect of the Developed Solution}

The project aimed at providing clinical researchers with an infrastructure to support the requirements of modern clinical trials. From data collection and integration, to workflow design and result analysis, initial studies in the project detected some major points of interest for the area. There were specific needs to cover to alleviate end-users from the most resource-consuming tasks in their daily work. The combination of thorough analysis of scenarios, research on previously proposed solutions and an extensive tool and service development led, after four years of work, to the completion of the p-Medicine Platform. Intensive testing within real-world scenarios provided highly promising results.

A novel personal health record (PHR) system was developed within the project, enabling patients to actively participate in the management of their disease, employing psycho-emotional questionnaires to monitor patients and to automatically give recommendations to their carers about their psycho-emotional status and optimal communication guidelines.

\subsubsection{Lessons Learnt}

The patient health records, and the diversity of data sources comprising these, make imperative the development of easy-to-use, standardized health informatics platforms. p-Medicine was designed to link pseudonymized patient data from multiple clinical sources, on which analytics and modelling tools may be applied. The flexible, distributed nature of the system makes it highly robust and scalable. The implementation of an e-consent scenario through pseudonymization enables, unlike many similar platforms, results from the analytic processes that are found to have an 
impact on an individual patient, to be directly communicated, via the trusted third party, to the clinicians treating the patient.

Central to the challenges addressed by the p-medicine was the issue of semantic interoperability between production systems in both the clinical word (Electronic Health Record (EHR) systems) and the research domain (Clinical Trial Management Systems, Clinical Report Forms (CRF) systems, etc.). As a result, the issue of standards became a central activity in the project. Standards relate to both technical aspects of the systems developed as well as terminology and semantic aspects of this work. It was the intention of the project to integrate concepts from existing standards, models and architectures, while extending and refining them where appropriate and required. In achieving this objective the project had a dedicated task focusing on standards. The activities involved monitoring of standards development, critical review and assessment of their applicability in the p-medicine framework, refining such standards based on domain specific requirements.

\subsection{EURECA}

The goal of the EURECA project was to enable seamless, secure, scalable and consistent linkage of healthcare information residing in EHR systems with information in clinical research information systems, such as clinical trial systems, supporting the two currently separated worlds of clinical research and clinical practice to connect and benefit from each other. EURECA objective was to build an advanced, standards-based and scalable semantic integration environment, enabling seamless, secure and consistent bi-directional linking of clinical research and clinical care.

\subsubsection{Technological Contribution}

The aim of EURECA was to provide a framework of tools which can be easily interconnected in different configurations, tailored to the needs of different environments and end-users. To obtain this high flexibility, loose coupling and a serviceorientated approach was chosen. The focus was thus on interoperability and interfacing in the architectural description. Modules and components designed and built within the project operated seamlessly through well-specified interfaces on different levels (i.e., interoperability on the level of IT-protocol, data format, information content, etc.). Figure 6.4 shows the architecture of the EURECA platform.

To obtain high flexibility, loose coupling and a service-orientated approach was chosen, emphasizing on interoperability and interfacing. Thus, EURECA architecture was determined by its interfaces. Modules and components designed and built within the project could operate together thanks to these well-specified interfaces at different levels. On the implementation level, EURECA internal services relied on SOAP as communication protocol. 


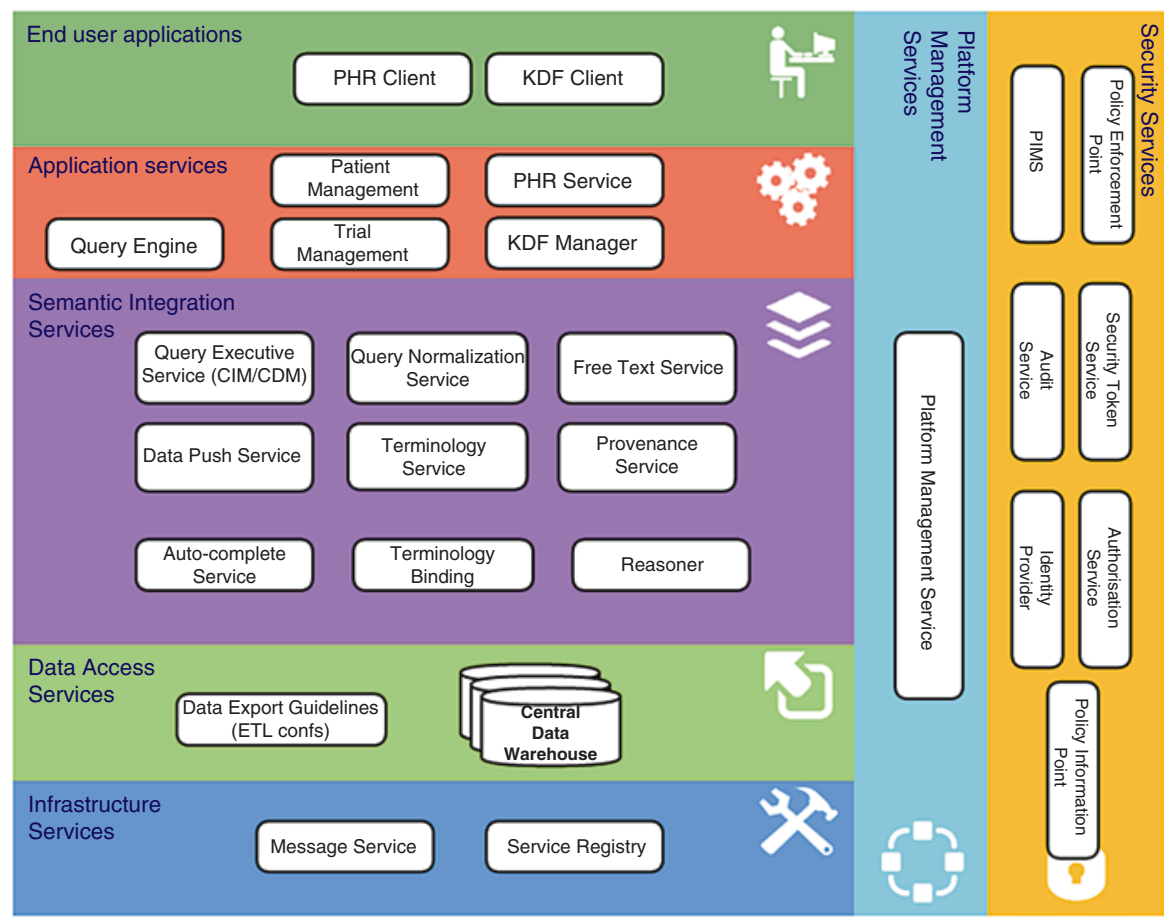

Fig. 6.4 The main components of the EURECA platform

The EURECA platform was designed as a multi-layered architecture, with responsibilities assigned to the various architectural layers. Every component designed within EURECA was mapped to one of these layers (or spanned over multiple layers). The architectural layers were:

- End-User Applications: The components situated in this layer could be seen as endpoints to the end-users of the system, presenting the underlying back-end functionality in an intuitive and user-friendly way. The components usually made use of a (advanced) graphical user interface (GUI) for displaying this (complex) back-end functionality.

- Application Services: This business layer provides the core functionality of the EURECA services as it houses a variety of application services. The components in this layer contained the functional algorithms that handled information exchange between the semantic integration layer and the presentation layer. Where possible, EURECA promoted the approach of providing re-usable services in the application layer.

- Semantic Integration Services: This layer utilized the ontology-based information model and translated or mapped the model to the underlying data and information sources. The semantic integration layer was abstracting the underlying data sources for the upper application layers. 
- Data Access Services: This layer contained the various data and the metadata repositories. Services on this layer were responsible for the actual data access. The data warehouses exposed standardized query interfaces, and queries were expressed using the EURECA core dataset.

- Infrastructure Services: The components placed in this layer provided service communication and service management capabilities to other EURECA components.

- Platform Management Services: Included components that were enabling the management of the integration of the various components in the EURECA platform.

- Security Services: On an orthogonal axis, the security layer was connected to all other architectural layers. The EURECA security solution consisted of re-usable modular components that respectively dealt with authentication, authorization, audit and privacy enhancing (i.e. services oriented specifically at data privacy protection).

\subsubsection{Participatory Aspects of the Developed Solution}

EURECA supported more effective and efficient execution of clinical research by: (i) Allowing faster eligible patient identification and enrolment in clinical trials, (ii) providing access to the large amounts of patient data, (iii) enabling long-term follow-up of patients, (iv) avoiding the need for multiple data entry in the various clinical care. In order to achieve the aforementioned goals, EURECA platform provided the clinical research access - in a legally compliant and secure manner - to the large amounts of patient data collected in the EHR systems to be used for new hypotheses building and testing (e.g. to benefit rare diseases), cohort studies, as well as protocol feasibility.

At the core of the project was the semantic interoperability among EHR and clinical trial systems, consistent with existing standards, while managing the various sources of heterogeneity: technology, medical vocabulary, language, etc. This required the definition of sound information models describing the EHR and the clinical trial systems, and capturing the semantics of the clinical terms by standard terminology systems. The scalability of the solution was achieved by modularization, identifying core data subsets covering the chosen clinical domains. The project demonstrated and validated concepts developed in EURECA by implementing a set of software services and tools that were deployed in the context of pilot demonstrators. EURECA developed solutions that fulfill the data protection and security needs and the legal, ethical and regulatory requirements related to linking research and EHR data.

\subsubsection{Lessons Learnt}

The main barriers of secondary use of EHR data for research and of enabling a consistent feedback loop to care are the lack of common technology standards and concept terminologies. While solving the interoperability issue in healthcare at the 
generic level is not a realistic approach (Boyle and Levin 2008), EURECA aimed at semantic interoperability on domains of concepts (i.e. describing specific clinical areas). It began from disease- and treatment-related sets of concepts in the oncology domain and demonstrated the proposed solution in concrete clinical scenarios. On top of the achieved semantic interoperability software services and tools to support more efficient research, better care and improved patient safety were developed.

The approach taken in EURECA was to rely when possible on existing initiatives and previous efforts in terminology development and standardization. The viability of the solutions was demonstrated and developed by implementing a set of loosely coupled interconnected services/modules that have been deployed in the context of several pilot demonstrators in the cancer area, at healthcare sites.

The EURECA environment aims to provide several software services that help to securely interconnect the clinical trial systems and the electronic health record systems. This will bring several benefits, among which are early detection of patient safety issues and more efficient recruitment of eligible patients. Consistent linkage between CTS and EHRs will also help to significantly reduce the need for double data entry, which is currently often common practice.

\subsection{INTEGRATE}

The FP7 INTEGRATE project (http://www.fp7-integrate.eu/) focused on the development of innovative biomedical applications for streamlining the execution of clinical research on cancer (Kondylakis et al. 2016). This was achieved by enabling multi-disciplinary collaboration, contributing to the management and the largescale sharing of multi-level heterogeneous datasets, and by developing new methodologies and predictive multi-scale models on cancer.

\subsubsection{Technological Contribution}

The technological infrastructure developed for the INTEGRATE project was composed of five layers as shown in Fig. 6.5.

On top, the presentation layer includes the various components that the end-users are using to access the patient screening application, the central pathology review, the cohort selection client and the analytical tools. The patient screening facilitates efficient identification of eligible patients for clinical trials through automatic matching of their characteristics and trial inclusion and exclusion criteria. Usually, patient data are described in free-text and this applies for trial exclusion and inclusion criteria. As such, automatic identification of eligible patients is a challenging task. On the other hand, enabling the collaboration and the participation of multiple clinical trial experts is an important challenge in modern multi-centric setting of clinical trials. The central pathology review tool enables high-quality, remote, decision making of multiple pathologists based on microscopy slides that are stored, 


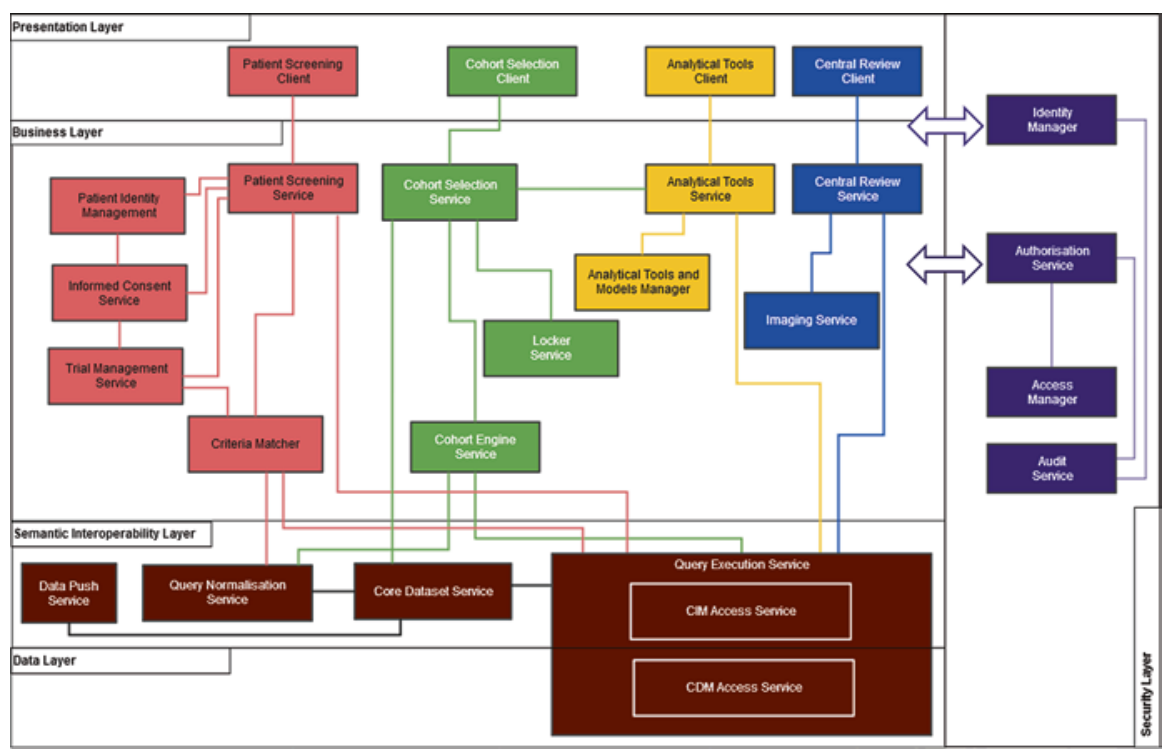

Fig. 6.5 A high-level view of the INTEGRATE technical Architecture (Kondylakis et al. 2016)

examined, annotated and commented online. Besides this, clinical researchers are able to formulate at run-time cohorts, based on multiple criteria available. In selected data can be visualized and analyzed in real time using the cohort selection and the analytical tools offered by the infrastructure.

Effective and efficient collaboration of clinical trial participants remains an important challenge in the modern multi-centric setting of such, post-genomic trials. The primary use of this Central Review for Pathology tool was to enhance the collaboration among groups of expert pathologists and to enable efficient, highquality decision making for patients participating in a clinical trial.

The business layer includes all necessary services for patient screening, cohort selection, analytical services, imaging services and central review services. The semantic interoperability layer provides access to the homogenized data through an ontology. As such, the data layer includes the various data sources along with their corresponding metadata. Finally, a security layer establishes all necessary services for authentication, authorization and identity management.

\subsubsection{Participatory Aspect of the Developed Solution}

Although the specific project did not focus on enhancing the participatory aspect of the patient, it is interesting to see that it was focusing on the participatory aspect of multiple experts involved in multi-centric clinical research and trials in cancer (oncologists, research and trial nurses, researchers, bioinformaticians, pathologists, trial coordinators). More specifically, the project developed ICT solution to improve 
the efficiency of clinical research and the data and knowledge flow between clinical research and clinical care and to enable the effective collaboration of all persons involved in the process. Evaluation performed showed that providing efficient and effective tools can be of high benefit in daily practice.

\subsubsection{Lessons Learnt}

Several lessons were learnt from this project, in the process of streamlining the execution of clinical research and to speed up the transfer of results to the clinical practice. A key lesson for example was that compliance to trial-related legislation, especially to the data protection laws, is a critical success factor for any research-network.

Next, effective and efficient graphical user interfaces are of utmost importance when dealing with domain experts who have no time to waste and usually show inertia when new methods and approaches are proposed to them. In this regard, a really important lesson is that providing useful applications with nice GUI is not enough. Those applications should be properly integrated with the ICT tools they are already using and with their daily routine. In addition, service performance and stability are two keys in workflows with multiple participants with limited time.

\section{Conclusions and Guidelines for Future Development}

This chapter presented experiences from five European research projects, all focusing on enabling patients and related stakeholders to actively participate effectively and efficiently in the journey of the patient. We described the technological solutions that were built, we focused on the participatory aspect of each individual project and we described relevant lessons learnt.

As described, transforming the recent model of paternalistic care to a participatory one has many challenges, especially in a chronic illness domain like cancer, with multiple participants and stakeholders involved in a journey that might span multiple years. Although ICT and the proliferation of portable devices have the potential to lead to a leap forward, the steps needed come with many challenges. This is being reflected by the high number of research projects currently focusing on promoting specifically this participatory aspect. Capitalizing on the presented lessons learnt we can summarize the following guidelines:

User-centred design: Technological tools should always involve end-users in all phases of the development in an iterative process and that usability is equally important to the effectiveness of the tools in order to gain user acceptance. Patient should not only be employed to use the final product, but should actively codesign the developed solutions, as eventually they are the ones to use them. 
Integration to daily workflow: In addition, in order to gain user acceptance the integration of the tools in the daily workflow of both the patients and the care providers is really important as they have limited time and they would like to minimize their potential involvements. Challenges related to semantic interoperability between production systems in both the clinical word (EHR systems) and the research domain (Clinical Trial Management Systems, CRF systems, etc.) should be resolved, whereas consistent linkage between clinical research and clinical practice will also help to significantly reduce the need for double data entry, which is currently often common practice. Specific attention should be paid to the fact that different professionals and organizations that participate in the patient's journey have different priorities (Schaller et al. 2016).

Mass scale: For long-term data collection of health-related data, individual participation at a mass scale is important. Such a comprehensive data collection will have a very strong clinical significance for diagnosis, prediction and individualized treatment, leading to significant evidence in health outcomes and quality of life.

Health psychology: Further involvement of the health psychology models will provide a foundation for health behaviour intervention, allowing the identification of key roles in patients' self-management, with a focus on their influence, and eventually on the compliance with medication plans, yielding individually tailored behaviour interventions.

Security and legal aspect: Securely interconnecting the clinical trial systems and the electronic health record systems can bring several benefits, among which are early detection of patient safety issues and more efficient recruitment of eligible patients. In addition, key challenges to be resolved are: assessing the validity of electronically given consent, data protection implications of access and use by third parties, the need for certification of apps/tools under the medical devices regime, and potential ex post facto liability in case of harm to users.

All these guidelines are essential, when creating ICT systems that are intended to be used by all involved participants in the journey of cancer management, but also for other chronic diseases as well, achieving eventually the P5 vision.

\section{References}

Barnato, A. E., Llewellyn-Thomas, H. A., Peters, E. M., Siminoff, L., Collins, E. D., \& Barry, M. J. (2007). Communication and decision making in cancer care: Setting research priorities for decision support/patients' decision aids. Medical Decision Making, 27(5), 626-634.

Bekker, H. L., Hewison, J., \& Thornton, J. G. (2003). Understanding why decision aidswork: Linking process with outcome. Patient Education and Counseling, 50(3), 323-329.

Boyle, P., \& Levin, B. (2008). World cancer report 2008. IARC Press, International Agency for Research on Cancer. 
Brown, R. F., et al. (2013). Perceptions of participation in a phase I, II, or III clinicaltrial among African American patients with cancer: what do refusers say? Journal of Oncology Practice/ American Society of Clinical Oncology, 9(6), 287-293.

Bucur, A., van Leeuwen, J., Christodoulou, N., Sigdel, K., Argyri, K., Koumakis, L., et al. (2016). Workflow-driven clinical decision support for personalized oncology. BMC Medical Informatics and Decision Making, 16(2), 87.

Davis, E. L., et al. (2014). An exploration of decision aid effectiveness: the im-pact ofpromoting affective vs: Deliberative processing on a health-related deci-sion. Health Expectations, 18, 2742-2752.

Engel, G. (1977). The need for a new medical model: A challenge for biomedical science. Science, 196, 126-129.

Faccio, F., Renzi, C., Crico, C., Kazantzaki, E., Kondylakis, H., Koumakis, L., et al. (2018). Development of an eHealth tool for cancer patients: monitoring psycho-emotional aspects with the Family Resilience (FaRe) Questionnaire. ecancermedicalscience, 12.

Frydman, G. (2010). A Patient-Centric Definition of Participatory Medicine. e-patients.net. Available online http://e-patients.net/archives/2010/04/a-patient-centric-definition-ofparticipatory-medicine.html, Accessed 20 Oct 2018.

Genitsaridi, I., Kondylakis, H., Koumakis, L., Marias, K., \& Tsiknakis, M. (2013). Towards intelligent personal health record systems: Review, criteria and extensions. Procedia Computer Science, 21, 327-334.

Genitsaridi, I., Kondylakis, H., Koumakis, L., Marias, K., \& Tsiknakis, M. (2015). Evaluation of personal health record systems through the lenses of EC research projects. Computers in Biology and Medicine, 59, 175-185.

Gorini, A., \& Pravettoni, G. (2011). P5 medicine: a plus for a personalized approach to oncology. Nature Review Clinical Oncology, 8(7), 444.

Gorini, A., Mazzocco, K., Gandini, S., Munzone, E., McVie, G., \& Pravettoni, G. (2014). Development and psychometric testing of a breast cancer patient profiling questionnaire. Journal of Breast Cancer: Targets and Therapy, 2014, 133-146.

Gravel, K., Légaré, F., \& Graham, I. D. (2006). Barriers and facilitators to implementing shared decision-making in clinical practice: A systematic review of health professionals' perceptions. Implementation Science, 1-16.

Hoffmann, S., \& Wilson, S. (2018). The role of serious games in the iManageCancer project. ecancermedicalscience, 12 .

Hood, L., \& Friend, S. H. (2011). Predictive, personalized, preventive, participative (P4) cancer medicine. Nature Reviews. Clinical Oncology, 8, 184-187.

Hood, L., Heath, J. R., Phelps, M. E., \& Lin, B. (2004). Systems biology and new technologies enable predictive and preventative medicine. Science, 306, 640-643.

Iatraki, G., Kondylakis, H., Koumakis, L., Chatzimina, M., Kazantzaki, E., Marias, K., \& Tsiknakis, M. (2018). Personal Health Information Recommender: implementing a tool for the empowerment of cancer patients. ecancermedicalscience, 12 .

Inerney, S. J. M. (2018). Introducing the biopsychosocial model for good medicine and good doctors. British Medical Journal, 324, 1533.

Internet World Stats. (2016). World Internet Users and 2016 Population Stats, Internet World Users on June 30, 2016. Miniwatts Marketing Group. http://www.internetworldstats.com/stats.htm. Accessed 30 Oct 2018.

Kane, H. L., et al. (2014). Implementing and evaluating shared decision making in oncology practice. CA: A Cancer Journal for Clinicians, 64(6), 377-388.

Kazantzaki, E., Kondylakis, H., Koumakis, L., Marias, K., Tsiknakis, M., Fioretti, C., \& Pravettoni, G. (2016). Psycho-emotional tools for better treatment adherence and therapeutic outcomes for cancer patients. pHealth, 2016, 129-134.

Keselman, A., \& Smith, C. A. (2012). A classification of errors in lay comprehension of medical documents. Journal of Biomedical Informatics, 45, 1151-1163. 
Kondylakis, H., Koumakis, L., Genitsaridi, E., Tsiknakis, M., Marias, K., Pravettoni, G., ... \& Mazzocco, K. (2012, November). IEmS: A collaborative environment for patient empowerment. Bioinformatics \& Bioengineering (BIBE), 535-540. IEEE.

Kondylakis, H., Koumakis, L., Kazantzaki, E., Chatzimina, M., Psaraki, M., Marias, K., \& Tsiknakis, M. (2015a). Patient empowerment through personal medical recommendations. In MedInfo, 1117.

Kondylakis, H., Spanakis, E. G., Sfakianakis, S., Sakkalis, V., Tsiknakis, M., Marias, K., Zhao, X., Yu, H.Q., Dong, F. (2015b). Digital patient: Personalized and translational data management through the MyHealthAvatar EU project. EMBC, 1397-1400.

Kondylakis, H., Flouris, G., Fundulaki, I., Papakonstantinou, V., \& Tsiknakis, M. (2015c). Flexible access to patient data through e-Consent. In Proceedings of the 5th EAI international conference on wireless mobile communication and healthcare (pp. 263-266). ICST (Institute for Computer Sciences, Social-Informatics and Telecommunications Engineering).

Kondylakis, H., Claerhout, B., Mehta, K., Koumakis, L., van Leeuwen, J., Marias, K., PérezRey, D., de Schepper, K., Tsiknakis, M., \& Bucur, A. I. D. (2016). The INTEGRATE project: Delivering solutions for efficient multi-centric clinical research and trials. Journal of Biomedical Informatics, 62, 32-47.

Kondylakis, H., Koumakis, L., Hänold, S., Nwankwo, I., Forgó, N., Marias, K., et al. (2017b). Donor's support tool: Enabling informed secondary use of patient's biomaterial and personal data. International Journal of Medical Informatics, 97, 282-292.

Kondylakis, H., Bucur, A., Dong, F., Renzi, C., Manfrinati, A., Graf, N., \& Tsiknakis, M. (2017a). iManagecancer: developing a platform for empowering patients and strengthening selfmanagement in cancer diseases. IEEE International Symposium on Computer-Based Medical Systems (CBMS), 2017, 755-760.

Koumakis, L., Kanterakis, A., Kartsaki, E., Chatzimina, M., Zervakis, M., Tsiknakis, M., et al. (2016a). MinePath: Mining for phenotype differential sub-paths in molecular pathways. PLoS Computational Biology, 12(11), e1005187.

Koumakis, L., Kondylakis, H., Chatzimina, M., Iatraki, G., Argyropaidas, P., Kazantzaki, E., Tsiknakis, M., Kiefer, S., Marias, K. (2016b). Designing smart analytical data services for a personal health framework. pHealth, 123-128.

Koumakis, L., Roussos, P., \& Potamias, G. (2017). minepath. org: A free interactive pathway analysis web server. Nucleic Acids Research, 45(W1), W116-W121.

Koumakis, L., Kondylakis, H., Katehakis, D. G., Iatraki, G., Argyropaidas, P., Hatzimina, M., \& Marias, K. (2018). A content-aware analytics framework for open health data. In Precision Medicine Powered by pHealth and Connected Health, 59-64.

Maniadi, E., Kondylakis, H., Spanakis, E., Spanakis, M., Tsiknakis, M., Marias, K., Dong, F. (2013). Designing a digital patient avatar in the context of the MyHealthAvatar project initiative. $B I B E, 1-4$.

Marés, J., Shamardin, L., Weiler, G., Anguita, A., Sfakianakis, S., Neri, E., ... \& Coveney, P. V. (2014). p-medicine: A medical informatics platform for integrated large scale heterogeneous patient data. In AMIA Annual Symposium Proceedings, 2014, 872.

Mehta, S., Hughes, N. P., Li, S., Jubb, A., Adams, R., Lord, S., et al. (2016). Radiogenomics monitoring in breast cancer identifies metabolism and immune checkpoints as early actionable mechanisms of resistance to anti-angiogenic treatment. eBioMedicine, 10, 109-116.

Morahan-Martin, J.M. (2004). How internet users find, evaluate, and use online health information: A cross-cultural review. Cyberpsychol Behav., 7, 497-10.

Potamias, G., Koumakis, L., \& Moustakis, V. (2005). Mining XML clinical data: The HealthObs system. Ingénierie des systèmes d'information, 10(1), 59-79.

Pravettoni, G., \& Gorini, A. (2011). A P5 cancer medicine approach: Why personalized medicine cannot ignore psychology. Journal of Evaluation in Clinical Practice, 17, 594-596.

Pravettoni, G., Cutica, I., Righetti, S., \& Mazzocco, K. (2016). Decisions and the involvement of cancer patient survivors: a moral imperative. Journal of Healthcare Leadership, 8, 121. 
Pravettoni, G., Mazzocco, K., Gorini, A., \& Curigliano, G. (2016b). Understanding cognitive processes behind acceptance or refusal of phase I trials. Critical Re-views in Oncology/ Hematology, 100, 69-73.

Schaller, S., Marinova-Schmidt, V., Setzer, M., Kondylakis, H., Griebel, L., Sedlmayr, M., et al. (2016). Usefulness of a tailored eHealth service for informal caregivers and professionals in the dementia treatment and care setting: the eHealthMonitor Dementia Portal. JMIR Research Protocols, 5(2).

Schera, F., Schäfer, M., Bucur, A., van Leeuwen, J., Ngantchjon, E. H., Graf, N., Kondylakis, H., Koumakis, L., Marias, K., \& Kiefer, S. (2018). iManageMyHealth and iSupportMyPatients: Mobile decision support and health management apps for cancer patients and their doctors. ecancermedicalscience, 12.

Sfakianaki, P., Koumakis, L., Sfakianakis, S., Iatraki, G., Zacharioudakis, G., Graf, N., Marias, K., \& Tsiknakis, M. (2015). Semantic biomedical resource discovery: A Natural Language Processing framework. BMC Medical Informatics and Decision Making, 15(1), 77.

Sfakianakis, S., Koumakis, L., Zacharioudakis, G., Tsiknakis, M. (2009). Web-based authoring and secure enactment of bioinformatics workflows. In Grid and Pervasive Computing Conference, $88-95$.

Sox, H. C., Higgins, M. C., Owens, D. K. (2013). Medical decision making.

Stacchiotti, S., \& Sommer, J. (2015). Building a global consensus approach to chordoma: A position paper from the medical and patient community. The Lancet Oncology, 16(2), e71-e83.

Stamatakos, G., Dionysiou, D., Lunzer, A., Belleman, R., Kolokotroni, E., Georgiadi, E., et al. (2014). The technologically integrated oncosimulator: Combining multiscale cancer modeling with information technology in the in silico oncology context. IEEE Journal of Biomedical and Health Informatics, 18(3), 840-854.

Van Schoors, M., Caes, L., Verhofstadt, L. L., Goubert, L., \& Alderfer, M. A. (2015). Systematic review: Family resilience after pediatric cancer diagnosis. Journal of Pediatric Psychology, 40(9), 856-868.

Von Bertalanffy, L. (2003). General system theory. In The science of synthesis: Exploring the social implications of general systems theory (p. 304). Boulder: University Press of Colorado.

Weston, A. D., \& Hood, L. (2004). Systems biology, proteomics, and the future of health care: Toward predictive, preventative, and personalized medicine. Journal of Proteome Research, 3, 179-196.

World Health Organization. [webpage on the Internet]. WHO definition of health. Available at https://www.who.int/about/mission/en/. Accessed October 20, 2018.

Zhang, X., Deng, Z., Parvinzamir, F., \& Dong, F. (2018). MyHealthAvatar lifestyle management support for cancer patients. ecancermedicalscience, 12.

Open Access This chapter is licensed under the terms of the Creative Commons Attribution 4.0 International License (http://creativecommons.org/licenses/by/4.0/), which permits use, sharing, adaptation, distribution and reproduction in any medium or format, as long as you give appropriate credit to the original author(s) and the source, provide a link to the Creative Commons license and indicate if changes were made.

The images or other third party material in this chapter are included in the chapter's Creative Commons license, unless indicated otherwise in a credit line to the material. If material is not included in the chapter's Creative Commons license and your intended use is not permitted by statutory regulation or exceeds the permitted use, you will need to obtain permission directly from the copyright holder.

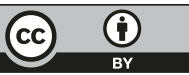

